\title{
Sexual orientation and suicidal behaviour in adolescents and young adults: systematic review and meta-analysis ${ }^{\dagger}$
}

A. Miranda-Mendizábal, * P. Castellví,* O. Parés-Badell, J. Almenara, I. Alonso, M. J. Blasco,

A. Cebrià, A. Gabilondo, M. Gili, C. Lagares, J. A. Piqueras, M. Roca, J. Rodríguez-Marín,

T. Rodríguez-Jiménez, V. Soto-Sanz, G. Vilagut and J. Alonso

\section{Background}

Research suggests that lesbian, gay and bisexual (LGB) adolescents have a higher risk of suicidal behaviours than their heterosexual peers, but little is known about specific risk factors.

\section{Aims \\ To assess sexual orientation as a risk factor for suicidal behaviours, and to identify other risk factors among LGB adolescents and young adults.}

\section{Method}

A systematic search was made of six databases up to June 2015, including a grey literature search. Population-based longitudinal studies considering non-clinical populations aged 12-26 years and assessing being LGB as a risk factor for suicidal behaviour compared with being heterosexual, or evaluating risk factors for suicidal behaviour within LGB populations, were included. Random effect models were used in meta-analysis.

\section{Results}

Sexual orientation was significantly associated with suicide attempts in adolescents and youths (OR $=2.26,95 \% \mathrm{Cl} 1.60$ 3.20). Gay or bisexual men were more likely to report suicide attempts compared with heterosexual men (OR $=2.21,95 \%$ Cl 1.21-4.04). Based on two studies, a non-significant positive association was found between depression and suicide attempts in LGB groups.

\section{Conclusions}

Sexual orientation is associated with a higher risk of suicide attempt in young people. Further research is needed to assess completed suicide, and specific risk factors affecting the LGB population.

\section{Declaration of interest}

None.

\section{Copyright and usage}

(c) The Royal College of Psychiatrists 2017.
Suicide is one of the leading causes of death and it represents a serious public health concern. Over the past 45 years worldwide suicide rates have increased by $60 \%$, the population of adolescents and youths registering the highest increase, ${ }^{1}$ making suicide the second cause of death in the group aged 15-29 years. ${ }^{2}$ There are few cases of attempted suicide before puberty, but a substantial increase occurs when individuals reach adolescence and young adulthood, especially between the ages of 19 and 23 years. In recent years studies have shown that lesbian, gay and bisexual (LGB) adolescents and youths have higher rates of suicidal ideation and suicide attempts than their heterosexual peers. ${ }^{3-5}$ It has been suggested that risk factors for suicidal behaviours differ between heterosexual and LGB groups, owing to the interaction of these markers with sexual orientation. ${ }^{6}$ Lesbian, gay and bisexual adolescents and youths are exposed to greater stigma, discrimination and victimisation within their families and romantic relationships compared with their heterosexual peers. ${ }^{7}$ Exposure to these factors may predispose these individuals to more mental health problems and suicide attempts. ${ }^{8,9}$ Minority stress theory, conceptualised as the burden of the perception of being different from others, ${ }^{10}$ may explain the higher levels of health risk behaviours in LGB youth, and is related to greater stigmatisation, marginalisation and a hostile social environment. ${ }^{11}$ In 2011 Marshal et al published a meta-analysis evaluating mental health in young LGB participants. ${ }^{12}$ Their results showed an estimated overall effect size for the association between sexual orientation and suicidality of $\mathrm{OR}=2.92$ (95\% CI $2.11-4.03$ ). However, that review was limited in several ways: first, suicide

*These authors contributed equally to the work.

†'see editorial, pp. 63-64, this issue. attempts were analysed as a single overall variable labelled 'suicidality', which included suicidal ideation, suicidal plan and suicide attempt without stratified analysis; second, the population age range was 18-25 years, lacking information on adolescents; third, the majority of the articles included in their meta-analysis were cross-sectional (only one was a cohort study); and finally only studies up to 2009 were taken into account.

For our review we adopted a more general and robust approach: suicide attempts and suicide were separated for the analyses, in order to assess associations that could be specific for these outcomes, and age range inclusion criteria were enhanced, rendering our results applicable to a wider population, consistent with international recommendations. ${ }^{13}$ Research published up to June 2015 was included, adding relevant recent information. More importantly, we assessed specific risk factors for suicidal behaviours and suicide death in the LGB population, thus contributing to filling a gap in knowledge about sexual minority groups in the adolescent and young adult populations, which have been little studied. An important characteristic of our review is that we included longitudinal studies only (either prospective cohorts or case-control studies). In fact, all except one of the articles analysed here were based on prospective cohort data. This ensured that exposure to the factors assessed preceded the outcome, making the evidence generated more relevant to establishing the temporal order of events, as well as minimising bias, improving the quality of included data and allowing us to establish valid and robust conclusions. ${ }^{14,15}$ There is still a need to improve understanding of suicidal behaviours of adolescent and young adult LGB populations, as well as to quantify more accurately the risk and protective factors for suicide. There is also a need to develop evidence-based public health strategies to 
reduce the prevalence of suicide attempts and suicide deaths among this high-risk population. We therefore undertook a systematic review of the literature, aiming to assess the extent to which sexual orientation is a risk factor for suicide attempts and suicide, and to identify risk factors for suicide attempts and suicide among LGB adolescents and young adults. The review was registered with the International Prospective Register of Systematic Reviews (PROSPERO; CRD42013005775). ${ }^{16}$

\section{Method}

Recommendations from the Meta-analysis of Observational Studies in Epidemiology (MOOSE) guidelines for systematic reviews, ${ }^{17}$ in relation to handling and reporting of results, were taken into account. Our initial search strategy was broad in scope and inclusive, with no restriction as to population or age, in order to identify predictors of suicidal-related behaviours. Text words, titles and $\mathrm{MeSH}$ terms were used as search terms, including suicide, suicidal behaviour, suicide attempt, suicidality, risk factor, causality, association, protective factor, incidence, longitudinal study, observational study, cohort study, case control study, prospective study, retrospective study, follow-up, and others, resulting initially in 23682 references after duplicates were removed. Detailed information about all the keywords used for inclusion and exclusion, and search terms used to identify suicide attempt, suicidal behaviour, population and study design are provided in online supplement DS1. The following databases were first searched up to October 2013 and updated June 2015 in order to include the most accurate data: Cochrane Library, Medline, PsycINFO, EMBASE and Web of Science. We searched grey literature using the OpenGrey European database, and reference lists from previous reviews and books were examined. No restriction of language or year of publication was applied. Corresponding authors for articles written in languages other than Spanish and English were contacted. For the broad-scope review, studies were included if they met all of the following criteria:

(a) reported suicide attempt or suicide as a dependent variable;

(b) assessed at least one risk factor for any of these outcomes;

(c) the study population age ranged between 12 years and 26 years, both inclusive;

(d) were population-based longitudinal studies (non-clinical and non-institutional sample cohorts) or case-control studies where the control group was of the same age range (non-clinical and non-institutional).

Using an expert consensus reported previously, suicide was defined as any act done with the intention of taking one's own life, whereas suicide attempt was defined as any act of self-injury with intention to die. ${ }^{18}$ Other suicide-related behaviours such as suicide ideation were excluded. Using these criteria we identified 197 studies for qualitative synthesis from the broader review. To these studies we applied the following specific selection criteria: first, studies that assessed LGB orientation as a risk factor for suicide attempts or suicide compared with a heterosexual peer group of the same age range; and second, studies that evaluated risk factors for suicide attempt or suicide within LGB populations. Studies assessing only the transsexual population were excluded, owing to certain conceptual differences. It has been reported that sexual orientation is a multidimensional concept referring to an enduring pattern of emotional, romantic and/or sexual attraction to males, females or both genders, ${ }^{10}$ whereas gender identity is one's own sense or conviction of maleness or femaleness. ${ }^{19}$ Moreover, transsexual individuals are considered to constitute a clinical population because transsexualism is classified as a type of gender identity disorder, ${ }^{20}$ and although this is a controversial issue, we are convinced that risk and protective factors may have different mechanisms of action and deserve further and specific research. Furthermore, during data synthesis, information corresponding to the transsexual population was not included.

\section{Study selection}

A multidisciplinary team of psychiatrists, psychologists, statisticians, epidemiologists and public health professionals was established to perform the review. Five groups of independent peer reviewers assessed all references. During the title review terms listed in online supplement DS1 were used, and discrepancies between reviewers were included. During the title and abstract review phases, reviewers were masked to the article's author, journal and year of publication to minimise selection bias. A third independent reviewer resolved any discrepancies during abstract and full-text review.

\section{Data extraction}

We adapted a Cochrane Collaboration data collection form for this review. Each reviewer extracted data and an independent reviewer examined the data entered in the form, checking that the information was entered properly and attempting to complete any missing data. In case of discrepancies, consensus among reviewers was established. For each article the following data were extracted: sample size; number of LGB participants included; age range; mean age; country of recruitment; study design; type of outcome (suicide death or attempt); type of sample recruited; and ethics committee approval. From cohort studies additional data were extracted: weeks of follow-up; number of suicide attempts during follow-up; and number of suicides during follow-up. Information about risk factors was obtained as odds ratios and 95\% confidence intervals; multivariate analysis prevailed over bivariate analysis. If available, stratified analysis was taken into consideration.

\section{Quality of studies}

The Newcastle-Ottawa Scale (NOS) was used for assessing the quality of non-randomised studies. ${ }^{21}$ This uses a 'star' system, in which a study is evaluated on three broad perspectives: the selection of the study groups, the comparability of the groups and the ascertainment of either the exposure or outcome of interest for case-control or cohort studies respectively. The scale consists of eight questions with different responses; the response indicating the highest quality is given a star. The highest-quality studies are awarded up to nine stars.

\section{Statistical analysis}

Suicide attempt and suicide were analysed comparing LGB and heterosexual groups. Additional analyses assessing specific risk factors of suicidal attempt and suicide within the LGB group were carried out. In the case of multiple publications of the same sample and predictive factors, results from the largest sample and longest follow-up were selected. Meta-analyses were performed for each variable for which there was a minimum of two studies with usable data; adjusted OR with 95\% CI were used when these data were provided in the articles; if not, unadjusted ORs were taken into account. Population attributable risk (PAR) was also calculated using the formula:

$$
\operatorname{PAR}=\frac{P(R R-1)}{(1+P(R R-1)}
$$

where $P$ is the prevalence of being LGB obtained through data from some of the cohort studies included in meta-analysis, ${ }^{22-27}$ 
and RR is the relative risk of suicide attempt in LGB $v$. heterosexual groups based on data from the cohort studies included. To convert the OR to RR the following formula was used:

$$
\mathrm{RR}=\frac{\mathrm{OR}}{\left(1-P_{0}\right)+P_{0} O R}
$$

where OR is the odds ratio of suicide attempt in LGB $v$. heterosexual groups and $P_{0}$ is the prevalence of suicide attempts in the heterosexual group, ${ }^{28}$ calculated through meta-analysis using data from four of the included articles. ${ }^{22-25}$ Stata software version 13 was used to conduct the meta-analysis.

Random effect methods were used for the meta-analysis. Statistical heterogeneity was assessed by visual inspection of forest plots, by Galbraith plots, by chi-squared tests to calculate $P$ value and by Higgins' test $\left(I^{2}\right)$, which describes the percentage of observed heterogeneity that would not be expected by chance. If $P$ was less than 0.10 heterogeneity was considered to be significant, and moderate if $I^{2}$ was $30-50 \%$ and severe if $I^{2}$ exceeded $50 \%{ }^{29}$ Small study effects (including publication bias) were assessed through visual inspection of funnel plots and Harbord's modification of Egger's test, which has been recommended for binary outcomes with effects measured as odds ratios. ${ }^{30}$ For fewer than three studies the Begg test was used. Sensitivity analyses were performed for cohort studies according to two criteria: first, length of follow-up corresponding to outcome assessment in the NOS score (less than 6 years), considering that the incidence of suicidal attempts and suicide is very low in non-clinical samples; and second, the country of origin of the samples, classified as USA $v$. non-USA. Because only three samples came from countries other than the USA, these could be a source of heterogeneity since there could be differences between countries in terms of social acceptance and stigma about sexual orientation.

\section{Results}

After duplicates were removed, 23682 articles were retrieved. After reviewing titles and abstracts the full text of 1575 potentially eligible articles were reviewed and 1561 were excluded for the following reasons: 397 were not population-based cohorts or case-control studies in which the control group was non-clinical; 436 did not involve participants aged 12-26 years; 183 did not concern LGB people or assess sexual orientation as a risk factor; 350 did not treat suicide or suicide attempt as a dependent variable; and 95 did not assess any risk factor (Fig. 1). After these exclusions a total of 14 articles were identified, including five articles that had not been reviewed by Marshal et al, ${ }^{12}$ even though the papers were published before 2009 and met their inclusion criteria. Of the final set of articles, ten assessed sexual orientation as a risk factor of suicide attempts or suicide, ${ }^{22-24,26,27,31-35}$ three assessed specific risk factors of these outcomes among LGB, ${ }^{36-38}$ and one assessed both sexual orientation as a risk factor and specific risk factors among LGB. ${ }^{25}$

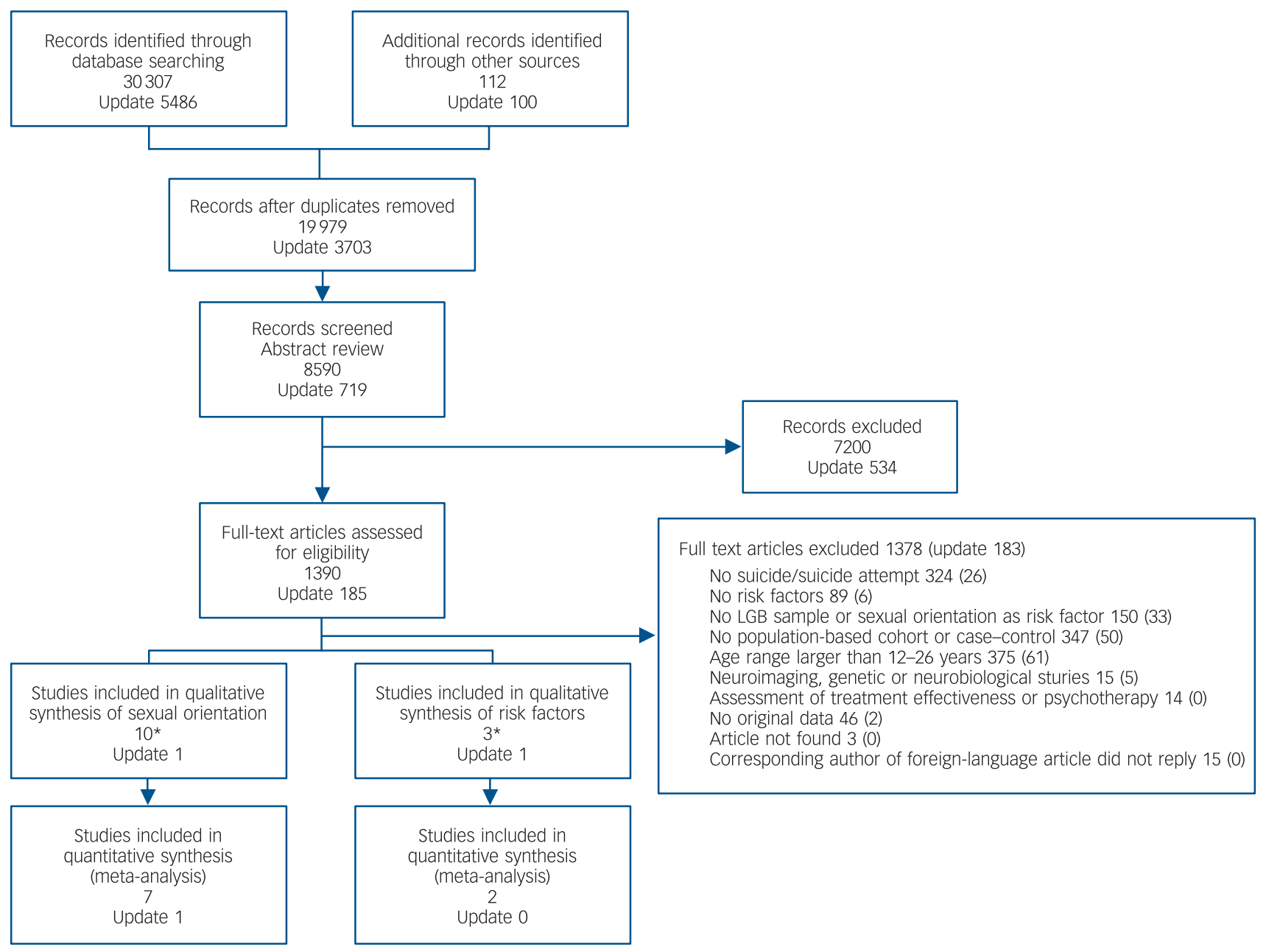

Fig. 1 Study selection. Studies in the qualitative synthesis included that by *Silenzio et al which assessed both sexual orientation as a risk factor, and risk factors in the lesbian, gay and bisexual population. ${ }^{25}$ 


\section{Sexual orientation as risk factor}

Eleven studies assessed LGB orientation as a risk factor for suicide attempt or suicide compared with heterosexual groups. These articles were published between 1995 and 2014, in four different countries: six from the USA, three from New Zealand, and one each from Norway and the UK (Table 1). Participation rates ranged from $30 \%$ to $100 \%{ }^{24,35}$ These studies contained data on 1634 LGB and 22117 heterosexual individuals and all of them included both male and female participants. Three studies were based on the general population, five recruited from high schools, one from a university and one among homeless adolescents. Based on data from seven of these articles, the prevalence of suicide attempts ranged from $1.5 \%$ to $3.5 \%$ in heterosexual adolescents and from $6 \%$ to $70 \%$ in LGB respondents. Ten studies assessed suicide attempt using a cohort design, ${ }^{22-27,31-33,35}$ resulting in a total population of 23484 , but only one study assessed suicide using a case-control design (cases $n=120$, controls $n=147$ ); Table $1 .^{34}$

\section{Quality of studies}

Of the 11 studies that assessed sexual orientation as a risk factor, no difference in quality was seen in selection domains in the cohort studies; in terms of comparability criteria eight articles were awarded two stars because relevant confounding factors were taken into consideration in the adjustment. ${ }^{22,24-27,31,32,37}$ However, different covariables were taken into account: one study considered demographic, psychosocial, history of mental illness, and formal and informal help-seeking variables; ${ }^{27}$ another included antecedents of suicidal behaviour; ${ }^{24}$ and yet another took into account variables related to parental characteristics. ${ }^{22}$ More detailed information is presented in Table 1. Regarding outcome domains, none of the studies achieved a star for ascertainment of outcome because data were self-reported by the participants and no confirmatory check of medical records was performed. The most significant difference was in length of follow-up, which was adequate only in five studies (Tables 2 and 3). ${ }^{22-24,26,31}$

\section{Sexual orientation and suicide attempts}

Three of the included studies reported a statistically significant higher risk for suicide attempt in LGB adolescents compared with a heterosexual group (adjusted OR 2.96-6.20), ${ }^{22,25,27}$ whereas three studies showed a non-significant increment of risk. ${ }^{26,32,35}$ Two articles presented rates of recent suicide attempts, values ranging from $5.4 \%$ to $17.6 \%$ for males and $2.1 \%$ to $15.5 \%$ for females. ${ }^{31,33}$ There were differences in terms of how sexual orientation was classified. Two studies used five categories: $100 \%$ heterosexual, mostly heterosexual, bisexual, mostly homosexual and $100 \%$ homosexual; ${ }^{27,33}$ four articles used only three categories: opposite-sex attraction only or heterosexual, minor same-sex attraction or bisexual, and persistent major same-sex attraction or homosexual; ${ }^{22,23,25,31}$ and the other studies used a single item to assess sexual orientation in two categories: LGB and non-LGB. ${ }^{24,26,32,35}$

Eight studies were included in the meta-analysis. Adjusted OR and $95 \%$ CI from results of each article were used. A single OR value for the whole population was not provided in two studies: Skegg et al presented values stratified by gender, ${ }^{23}$ and Whitlock et al reported OR for three sexual orientation categories, ${ }^{27}$ resulting in eleven samples included for meta-analysis. Three studies were excluded because data were either non-extractable or in a format that did not permit comparisons with the other studies. Random effect models were used because severe heterogeneity was observed $\left(I^{2}=60 \%, P=0.005\right)$. The overall pooled estimate in meta-analysis of cohort studies of suicide attempt showed a significantly higher risk for LGB youths when compared with heterosexual youths $(\mathrm{OR}=2.32$, 95\% CI 1.59-3.39). Two studies appeared to be sources of heterogeneity: one assessed sexual orientation through three different categories, ${ }^{27}$ whereas in the other the LGB sample size was only 28 but there were 979 in the heterosexual group. ${ }^{22}$ Furthermore, on the Galbraith plot, these two studies were outliers. After exclusion of these two studies the new OR was 2.26 (95\% CI $1.60-3.20 ; I^{2}=35 \%, P=0.161$ ). Visual inspection of the funnel plot does not suggest any publication bias, as small studies tend to lead to lower estimates of the effect than larger studies (Fig. 2). Although the Harbord test was not significant $(P=0.4)$ it is important to note that these results should be interpreted with caution, since the small number of studies means there is insufficient power to distinguish chance from real asymmetry.

\section{Sensitivity analyses}

Taking into consideration only the studies that did not appear as possible sources of heterogeneity, sensitivity analyses were carried out according to length of follow-up and country of origin of the samples. Removing three studies with insufficient length of follow-up, results were not altered substantially with respect to the association between sexual orientation and suicide attempt $(\mathrm{OR}=2.59,95 \%$ CI 1.58-4.26), whereas heterogeneity decreased compared with the first meta-analysis $\left(I^{2}=45 \%, P=0.122\right)$. Possible explanations for observed heterogeneity could be the differences among covariables used for the adjustment, and gender differences because one study reported results for female participants,${ }^{24}$ another for males and females, ${ }^{23}$ and two studies presented a single value for both genders together. ${ }^{25,26}$ Similar results were obtained when samples from the USA were taken into account in the analyses, i.e. an increased risk in LGB youths $(\mathrm{OR}=1.98,95 \%$ CI $1.42-2.75)$ with no heterogeneity $(P=0.488)$.

\section{Gender stratification}

According to one study the lifetime prevalence of suicide attempts in gay and bisexual men was $4.8 \%$, but $12.6 \%$ in lesbian and bisexual women in the same sample. ${ }^{23}$ Only two cohort studies assessed sexual orientation as a risk factor of suicide attempts stratified according to gender, ${ }^{23,32}$ and one study presented results only for women. ${ }^{24}$ One of these articles found a significantly increased risk for lifetime suicide attempts in gay and bisexual men compared with heterosexual men $(\mathrm{OR}=3.2,95 \%$ CI $1.4-$ $7.2){ }^{23}$ Similar results were found for the risk of lifetime suicide attempts among lesbian and bisexual women; one study reported a risk almost five times higher $(\mathrm{OR}=4.96,95 \% \mathrm{CI} 2.29-10.62){ }^{24}$ In the other studies results in men showed that homosexual orientation was not associated with higher risk $(\mathrm{OR}=1.71,95 \%$ CI $0.92-3.17){ }^{32}$ findings were similar in women: $\mathrm{OR}=1.4(95 \%$ CI $0.7-2.7),{ }^{23}$ and OR $=1.25(95 \%$ CI $0.80-1.96){ }^{32}$

Meta-analysis found an increased risk of suicide attempts in gay or bisexual men compared with heterosexual men $(\mathrm{OR}=2.21$, 95\% CI 1.21-4.04) (online Fig. DS1(a)). The Galbraith plot showed that the studies fell within confidence limits (online Fig. DS1(b)) and no publication bias was observed (Begg test, $P=0.317$ ) (online Fig. DS1(c)). Being a lesbian or bisexual woman was associated with higher risk of suicide attempts, but this was not significant $(\mathrm{OR}=1.97,95 \%$ CI $0.90-4.30)$ (online Fig. DS2(a) ) and severe heterogeneity was observed $\left(I^{2}=79 \%\right.$, $P=0.008)$. In the Galbraith plot one study appeared as a cause of variability (online Fig. DS2(b)); ${ }^{24}$ after excluding this study the OR was 1.29 (95\% CI 0.89-1.88). As in the analyses for 


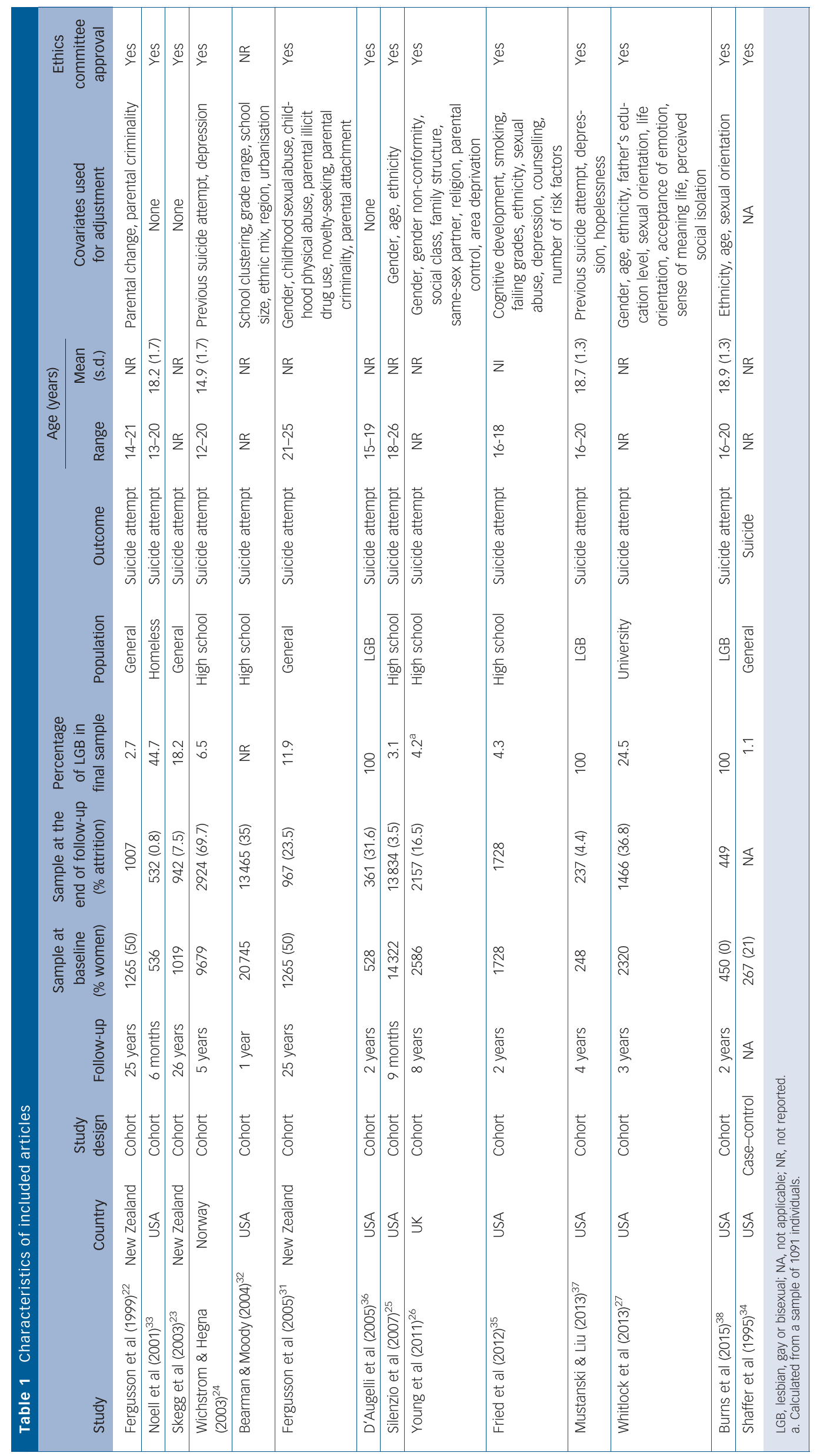




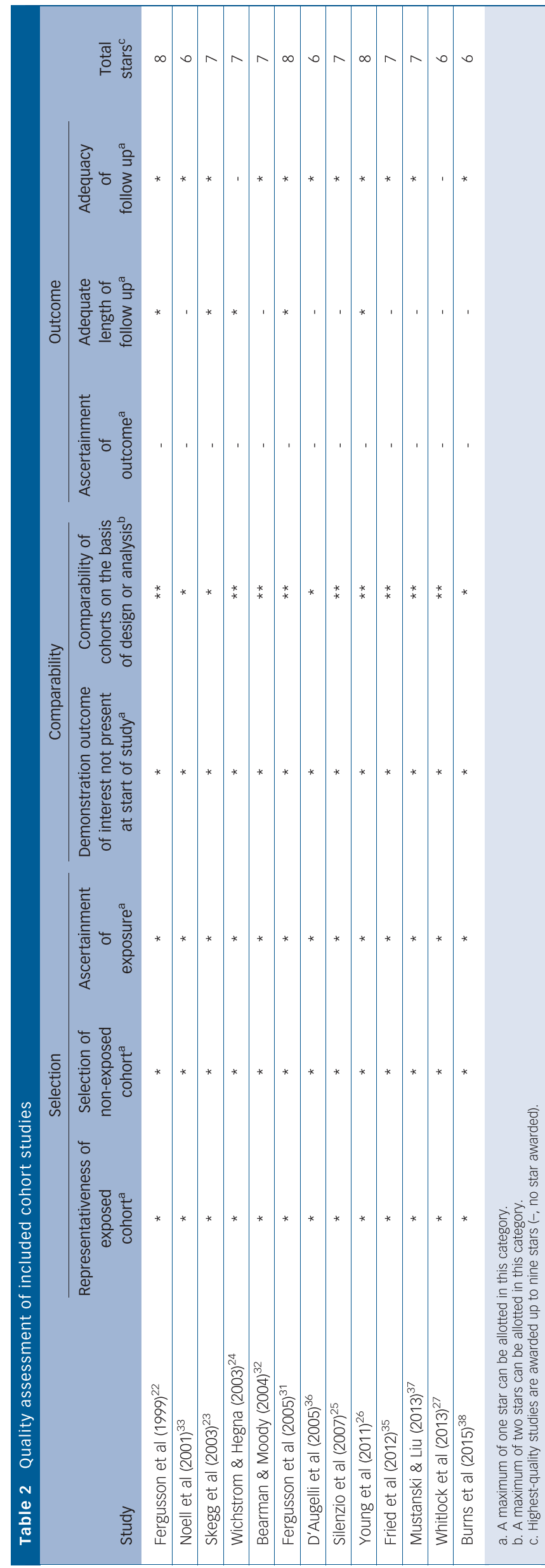

men, no publication bias was observed (Begg test, $P=0.117$ ) (online Fig. DS2(c)). Meta-analysis comparing gay with heterosexual men showed significantly increased risk $(\mathrm{OR}=8.36$, 95\% CI 1.88-37.11) (online Fig. DS3(a)); a similar situation occurred when bisexual men were compared with heterosexual men $(\mathrm{OR}=2.44,95 \%$ CI 1.16-5.16) (online Fig. DS(3)b). The results obtained in meta-analysis of lesbian $v$. heterosexual women demonstrated increased risk of suicide attempt $(\mathrm{OR}=4.31,95 \%$ CI 1.89-9.86) (online Fig. DS3(c)); and also in bisexual $v$. heterosexual women, although not significant $(\mathrm{OR}=1.56,95 \%$ CI 0.66-3.69) (online Fig. DS3(d)).

\section{Sexual orientation and suicide}

Only one case-control psychological autopsy study evaluated completed suicide. The objective was to assess risk profiles of all people who died by suicide under 20 years of age, through interviews with their parents (or other caregiver at the time of the death), a sibling or a friend - in one case a schoolteacher who had known about the person's behaviour was interviewed. Cases were suicide deaths, controls were a random sample from telephone subscribers in the study area. Out of 120 cases that were completely investigated $79 \%$ of the individuals were male, of whom 3 were gay. No one in the control group reported any homosexual experience. Even though no odds ratio value was reported, it was stated that the difference was not significant $(P=0.088)$. None of the women who died by suicide was lesbian. ${ }^{34}$

\section{Population attributable risk}

Using different values of the prevalence of being LGB corresponding to different scenarios ( $8 \%$, range 4-13) and assuming the relationship between sexual orientation and suicide attempt is causal and not confounded ( $\mathrm{RR}=2.15,95 \%$ CI 1.56-2.94), calculations suggest that $8 \%$ (range 4-13) of suicide attempts in adolescents and young adults could be attributed to sexual orientation (see online Table DS1).

\section{Risk factors}

Four studies that assessed specific risk factors for suicide attempts among the LGB population were identified (but none assessing suicide). These studies took place in the USA and were published in 2005 and $2015 .^{25,36-38}$ Participation rates ranged between almost $70 \%$ to $99.7 \%{ }^{36,38}$ All of them used a cohort design and together contained data on 1476 LGB people, with representation of both genders (Table 1).

\section{Quality of studies}

According to our quality assessment, no difference was seen in selection domains between the four studies. Sample sizes ranged from $n=20$ to $n=449$; one study analysed data from a nationally representative sample of adolescents and young adults, ${ }^{25}$ whereas the other three studies recruited participants from different US cities. ${ }^{36-38}$ In the comparability domain, two studies achieved two stars because important confounders were taken into account in the design and/or the analysis. Two studies considered age and ethnicity as covariables for the analysis, ${ }^{25,37,38}$ whereas Burns et al also included sexual orientation. ${ }^{38}$ The other study considered only age during the recruitment. ${ }^{36}$ For the ascertainment of the outcome, none of the studies had a star because suicide attempts were self-reported by the participants and no reference to records had been done to confirm. In terms of length of follow-up, none of the studies achieved a star; however, in the category that assessed the adequacy of follow-up the four articles had the 


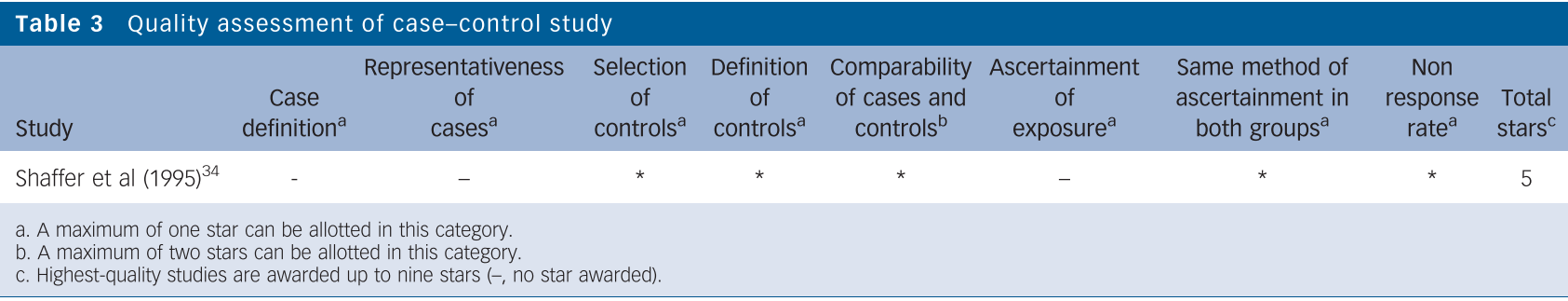

highest rating because attrition during the study was not related to the exposure or the assessed outcome. In general, all these studies had high overall quality (see Table 2 ).

\section{Data syntheses}

D'Augelli et al evaluated an important number of risk factors for suicide attempts; results showed that 61 individuals of the entire sample $(n=361)$ of LGB youths made a suicide attempt. ${ }^{36}$ Risk factors such as having been more open about being LGB with their families, more often called 'sissy' or 'tomboy' by parents, more gender atypical in childhood, having experienced parental psychological abuse and a family history of depression or suicidality all presented higher prevalence or median values. Mustanski \& Liu reported that having a history of suicide attempts represented an increased risk of subsequent suicide attempt whereas hopelessness and depression were not significant. ${ }^{37}$ The results obtained by Silenzio et al indicated that problem drinking, depression and problem drug use were not related to increased risk of suicide attempts in LGB respondents, contrary to findings in non-LGB participants. ${ }^{25}$ Finally, results from the USA reported by Burns et al suggest that in some young LGB males from minority ethnic groups such as native Americans there is a 3-fold risk of suicide attempts compared with young White LGB men. ${ }^{38}$ However, Black or Hispanic men do not seem to have higher risk. More details of the risk factors assessed are presented in online Table DS2. Meta-analysis of depression as a risk factor for suicide attempts in LGB adolescents showed increased risk $\left(\mathrm{OR}=1.05,95 \%\right.$ CI $\left.0.93-1.19 ; \quad I^{2}=38 \%, \quad P=0.204\right) \quad$ (online Fig. DS4(a)), but the result was not significant. Using a Galbraith plot no heterogeneity was observed (online Fig. DS4(b)). No publication bias was found, according to the Begg test $(P=0.317)$ (online Fig. DS4(c)).

\section{Discussion}

Our systematic review shows that sexual orientation is significantly associated with suicide attempts, based on metaanalysis of longitudinal studies. Nevertheless, not enough studies were found to associate sexual orientation with suicide. Sexual minority men were more likely to make suicide attempts than

(a)

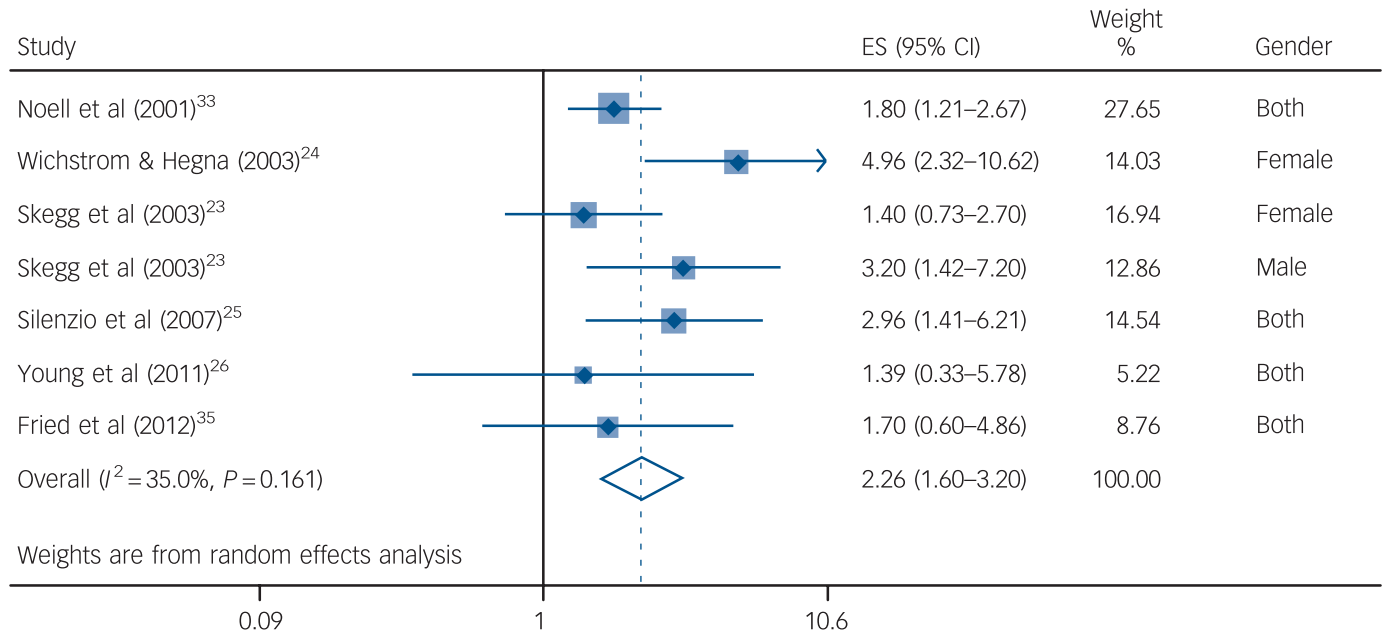

(b)

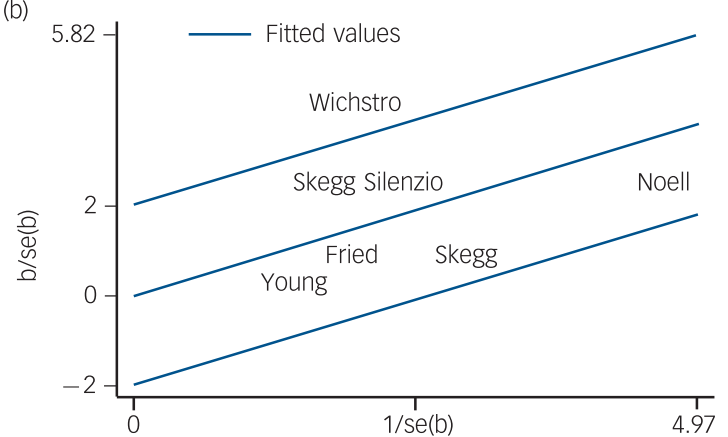

(c)

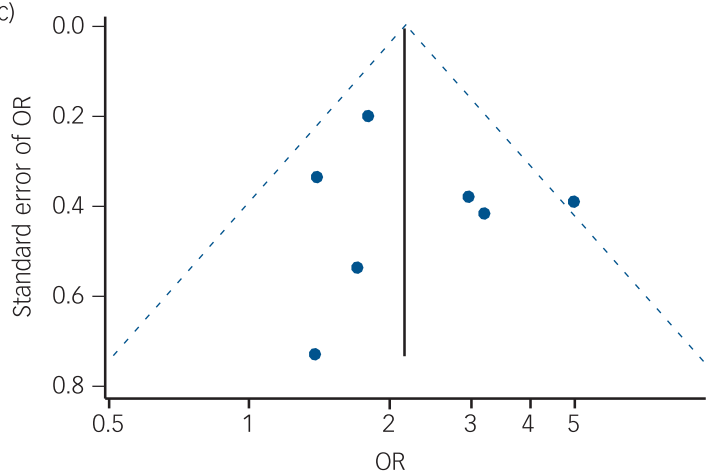

Fig. 2 Sexual orientation as risk factor for suicide attempts: (a) forest plot; (b) Galbraith plot; (c) funnel plot (Harbord test $P=0.43$ ). ES, effect size. 
heterosexual men. Among women, a similar association was found but it did not reach statistical significance, probably owing to the small number of studies assessed. Few studies were found evaluating risk factors for either suicide attempts or suicide among LGB populations. Further research assessing specific risk factors is needed.

\section{Strengths and limitations}

Our systematic review has several strengths: a broad-scope search in several databases and different languages was conducted, with peer review in the screening phase, independent review in the data extraction phase and use of methods for minimising bias; corresponding authors for articles written in languages other than Spanish and English were contacted for further information; a manual search and grey literature search were also carried out; and as far as we are aware no PAR calculation of sexual orientation as a risk factor for suicide attempts has been done in previous systematic reviews.

In relation to a previous systematic review, ${ }^{12}$ our study extended the search strategy to three additional databases and a grey literature search was also performed; we used a wider age range as an inclusion criterion, considering adolescents, and also included studies published after 2009. This resulted in 13 additional studies, 5 of which were considered for meta-analysis, adding new, relevant and useful information for the assessment of causes and mechanisms, despite our having excluded other suicide-related outcomes, such as suicidal ideation and plan, and cross-sectional studies, which had been included in the previous review. ${ }^{12}$ These additional articles added relevant and useful new information about the risk of sexual minority groups for future suicidal behaviours and death. Moreover, our results suggest the need for further assessment about the causes and mechanisms of suicidal behaviours and death among this population. A limitation is that our search strategy was not applied to the CINAHL and Sociological Abstracts databases. Additional papers might have been retrieved if these databases had been searched; however, we searched the databases most recommended for psychiatric research, including Web of Science and PsycINFO, ${ }^{39}$ and also performed a manual search. Further, we searched grey literature only in OpenGrey, a European database; although it includes access to Greynet data (an international grey literature database) there is no certainty that our search was exhaustive. Overall, the use of the six particular databases in this study is in line with most strict systematic reviews and provides important coverage security. An additional limitation of our review is that the articles included came from a broader search strategy, as has been done in other systematic reviews. ${ }^{40-42}$ However, in accordance with the initial inclusion criteria, both 'LGB population' and 'sexual orientation' as a risk factor were included in the search phase. Also, we included population-based studies in our review, which could have resulted in a higher heterogeneity. However, after excluding studies that seemed to be sources of heterogeneity, OR values became similar. Finally, we used the Newcastle-Ottawa Scale to assess the quality of the studies reviewed because it had been used in previous systematic reviews, ${ }^{43-46}$ and also because it was designed for longitudinal studies. However, evidence about its validity is still limited. ${ }^{21}$

\section{Comparison with other studies}

Consistent with other reviews, sexual minority adolescents and youths were more likely to have made suicide attempts than their heterosexual peers. We estimated a 2 -fold risk, which is similar to the risk ratios reported by King et al, which ranged from 1.96 to 2.76 in 12 -month prevalence of suicide attempts, for LGB population of any age range. ${ }^{47}$ Also, Marshal et al showed a risk of 2.92 in LGB young people aged from 18 to 25 years, ${ }^{12}$ slightly higher than our results, probably because most of the included studies were cross-sectional and their OR values ranged from 1 to 10 (ours ranged from 1.30 to 5). These facts may lead to overestimation of the risk; however, this must be interpreted with caution. We estimated the risk of suicide attempts according to sexual orientation, stratifying by gender. Our results are consistent with previous original studies which stated that being a gay or bisexual male is associated with higher risk of suicide attempt than being a heterosexual male. Longitudinal studies have found that sexual orientation is an independent risk factor for suicide attempts among young males, more so than among females. ${ }^{48}$ This finding could be related to results from other studies where elevated rates of suicide attempts in gay and bisexual adolescent men have been reported. ${ }^{49-52}$ During adolescence models of gender, masculinity and femininity are reinforced; a possible explanation for observed data could be that during this stage of life these models may be affected by heteronormativity. It is important to note that gender definition covers aspects related to social categories and different life spheres, including sexual facets. Heterosexuality or homosexuality must be understood only as forms of sexual expression, since there are interrelations between non-sexual and sexual spheres. ${ }^{53}$ In adolescents, the self-perception of failure to conform with this kind of model may affect different aspects of mental health. ${ }^{54}$

Among lesbian or bisexual women an increased risk of suicide attempt was found; however, it was not statistically significant, probably because few studies were identified. Our results also indicated that being bisexual is associated with higher risk of suicide attempt, but not in women; again, a possible reason could be the small number of studies. A previous systematic review summarised available research about bisexuality as a risk factor; it was associated with suicidal behaviour to a greater extent than heterosexuality. ${ }^{55}$ There is no clear explanation or identification of specific risk factors acting among those with bisexual orientation. It seems therefore plausible that the same risks and mechanisms such as more psychological distress and mental health problems (bisexuality and suicide) - act among all LGB categories.

Few studies were identified that evaluated risk factors for suicide attempts and suicide in LGB adolescents and youths. As a consequence, we could only perform a meta-analysis for the role of depression. According to our results depression does not seem to be related to suicide attempts in LGB population-based studies, in contrast to reports for the heterosexual population; however, these results should be interpreted with caution, because the lack of effect may be due to the insufficient number of included studies. Some authors suggest that minority stress theory may explain differences between the mechanisms of action of risk factors in the LGB population compared with the heterosexual population. This theory shows how specific external stressors such as victimisation, discrimination or stigma, or an internal stressor such as internalised homophobia, could increment suicide and suicidal behaviour risk. Discrimination involves anti-gay behaviour, including rejection, acts of physical violence and verbal assaults against gay men and other sexual minorities based on actual or assumed sexual orientation. ${ }^{56}$ Internalised homophobia refers to internalised societal homophobic attitudes, ${ }^{57}$ and includes negative attitudes toward homosexuality, displeasure with sexual orientation of others, disconnectedness from other LGB individuals, and discomfort with same-sex sexual activity. ${ }^{58}$

Negative 'coming out' reactions from family and friends, the experience of sexuality-oriented victimisation and having used drugs or alcohol to confront problems relating to their lesbian 
or gay identity increase the risk of attempted suicide in adolescents who discover their same-sex preference early in adolescence. ${ }^{59}$ Parental intolerance and rejection in response to the disclosure of an adolescent's sexual orientation, considered as forms of discrimination, are associated with specific risks, including depression, suicidal ideation, isolation, homelessness, prostitution, substance use, unprotected sex and sexually transmitted disease. ${ }^{59-61}$ Finally, internalised homophobia was found to be significantly related to psychological distress: guilt, sex difficulties, suicide (ideation and/or behaviour) and AIDS-related traumatic stress response. Other factors such as stigma and experience of prejudice events were also significantly related to most of the measures of distress; however, internalised homophobia was reliably the most potent predictor. The interaction between these three factors causes psychological maladjustment, known as 'psychologically injurious effects.'

Lesbian, gay and bisexual adolescents and young adults present significant differences compared with their heterosexual peers in relation to mechanisms by which risk factors act and are correlated. Risk factors such as depression, alcohol and drug misuse may be similar among LGB and heterosexual young people, but the mechanisms of action are completely different.

\section{Generalisation of the findings}

Some additional issues should be taken into consideration before generalising these results. First, the assessment of sexual orientation differed between included studies: one study assessed sexual orientation by asking if the person had ever had a sexual relation with another person of the same gender; this could be considered a sexual behaviour rather than sexual orientation. ${ }^{62}$ It is important to differentiate sexual orientation from sexual behaviour. Sexual orientation is composed of emotional, romantic and/or sexual attraction, as well as an individual's sense of personal and social identity. ${ }^{63} \mathrm{~A}$ person who has engaged in homosexual or bisexual behaviour should not necessarily be identified as lesbian, gay or bisexual. Second, we found important differences in the inclusion of confounding variables in multivariate models between the original studies. Some of them did not even adjust for any confounding variable, an important source of heterogeneity. Third, the length of follow-up in most of the cohort studies included was less than 5 years. Considering that the incidence of suicidal behaviour in the general population is low, ${ }^{2}$ it is important to take latency into account because studies allowing for latent periods found higher incidence of the outcomes than those that ignored latency. ${ }^{17}$ However, apparently this issue did not result in heterogeneity, as may be seen in the sensitivity analyses. Finally, although no publication bias was observed according to the Harbord test, we cannot firmly exclude it owing to the small number of studies analysed, well below the overall recommendation of a minimum of 10 studies for the assessment of asymmetry. ${ }^{64}$ Even though the funnel plot should be interpreted with caution owing to the small sample size and the problems previously reported when applied to a binary outcome, ${ }^{64}$ visual inspection of the graph suggests that if any, asymmetry would be in the sense of small studies tending to lead to lower estimates of the effect than larger studies, and thus publication bias does not seem to be explaining the asymmetry. ${ }^{65}$

\section{Future research}

Although a significant association between sexual orientation and suicide attempt has been established, research is needed in several areas. Sexual orientation and gender identity ought to be measured in a homogeneous way, or using the same definition by expert consensus, to allow comparisons between studies. Information about sexual orientation could be obtained from national surveys or registers, and there is a need for an appropriate method to determine the sexual orientation of people who have died by suicide. Longitudinal studies are needed to assess mediators such as victimisation, stigmatisation and discrimination to identify causal pathways between sexual orientation and suicidal behaviour. Public health prevention strategies ought to be developed that could reduce suicide attempts by around $9 \%$, according to our PAR calculations, which indicated that sexual orientation is a major contributor to suicidal behaviour. Inclusion of LGB participants should be encouraged in research about effective public health strategies to reduce risk factors and suicidal behaviour among the LGB population, and research should be extended to other settings, such as developing countries. Sexual orientation is associated with increased risk of suicide attempts in LGB adolescents and young adults, and gay and bisexual men are more likely to make suicide attempts; this is probably the case in women as well but our results were inconclusive. Further research is needed to establish causality between sexual orientation and suicide, and specific risk factors among the LGB population. Public health strategies for prevention of mental disorders including suicidal behaviour must be considered among the LGB population, a high-risk group in which specific factors are acting.

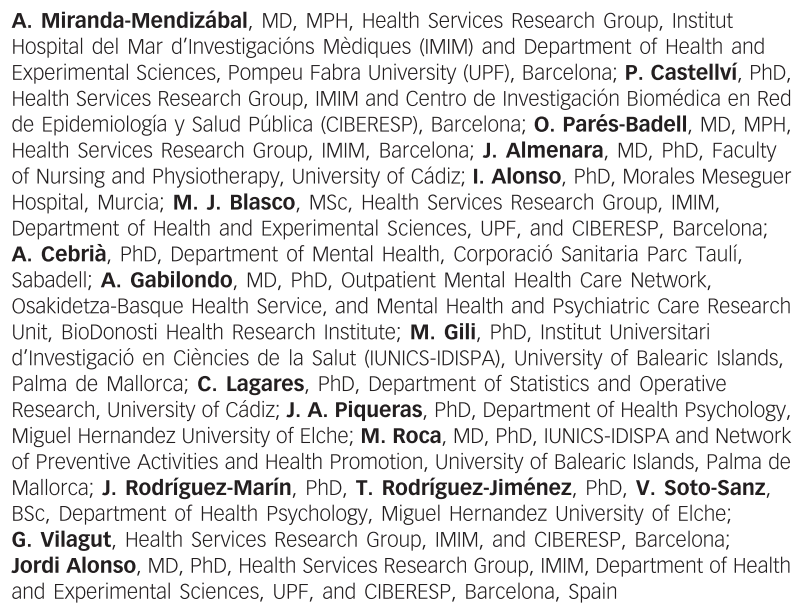

Correspondence: Dr Jordi Alonso, IMIM-Institut Hospital del Mar

d'Investigacions Mèdiques, PRBB Building, Doctor Aiguader 88, 08003 Barcelona, Spain. Email: jalonso@imim.es

First received 11 Feb 2016, final revision 21 Nov 2016, accepted 27 Nov 2016

\section{Funding}

A.M.M. was fully supported by the Secretaria Nacional de Educación Superior, Ciencia, Tecnología e Innovación, Ecuador. Additional funding for this study was provided by Instituto de Salud Carlos III (CD12/00440), ISCIII-FEDER (PI13/00343), ISCIII-FIS (CM14/ 00125), ISCIII ECA07/059 and AGAUR 2014 SGR 748, Spain.

\section{Acknowledgements}

Special acknowledgements are due to Dave MacFarlane for his assistance with management of the data extraction form, and to Alejandro De La Torre for his cooperation in statistical analyses.

\section{References}

1 World Health Organization Europe. Mental Health: Facing the Challenges, Building Solutions. WHO, 2005. 
2 World Health Organization. Preventing Suicide, A Global Imperative. WHO 2014

3 Borges G, Angst J, Nock MK, Ruscio AM, Kessler RC. Risk factors for the incidence and persistence of suicide-related outcomes: a 10-year follow-up study using the National Comorbidity Surveys. J Affect Disord 2008; 105 : 25-33.

4 Kessler R, Berglund P, Borges G. Trends in suicide ideation, plans, gestures, and attempts in the United States, 1990-1992 to 2001-2003. JAMA 2005; 293 1990-2.

5 Brent $D$, Mann J. Family genetic studies, suicide, and suicidal behavior. Am J Med Genet C Semin Med Genet 2005; 133C: 13-24.

6 Remafedi G, French S. The relationship between suicide risk and sexual orientation: results of a population-based study. Am J Public Health 1998; 88 57-61.

7 Haas AP, Eliason M, Mays VM, Mathy RM, Cochran SD, D'Augelli AR, et al. Suicide and suicide risk in lesbian, gay, bisexual, and transgender populations: review and recommendations. J Homosex 2011; 58: 10-51.

8 McLaughlin KA, Hatzenbuehler ML, Keyes KM. Responses to discrimination and psychiatric disorders among Black, Hispanic, female, and lesbian, gay, and bisexual individuals. Am J Public Health 2010; 100: 1477-84.

9 Díaz R, Ayala G, Bein E, Henne J, Marin B. The impact of homophobia, poverty, and racism on the mental health of gay and bisexual Latino men: findings from 3 US cities. Am J Public Health 2001; 91: 927-32.

10 Coker TR, Austin SB, Schuster MA. The health and health care of lesbian, gay, and bisexual adolescents. Annu Rev Public Health 2010; 31: 457-77.

11 Meyer I. Prejudice, social stress, and mental health in lesbian, gay, and bisexual populations: conceptual issues and research evidence. Psychol Bull 2003; 129: 674-97.

12 Marshal M, Dietz L, Friedman M. Suicidality and depression disparities between sexual minority and heterosexual youth: a meta-analytic review. $J$ Adolesc Health 2011; 49: 115-23.

13 World Health Organization. Health for the Worlds Adolescents. A Second Chance in the Second Decade. WHO, 2014.

14 Garg AX, Hackam D, Tonelli M. Systematic review and meta-analysis: when one study is just not enough. Clin J Am Soc Nephrol 2008; 3: 253-60.

15 Tooth L, Ware R, Bain C, Purdie DM, Dobson A. Quality of reporting of observational longitudinal research. Am J Epidemiol 2005; 161: 280-8.

16 Alonso J, Castellvi P, Pares O, Gabilondo A, Cebria Al, Rodriguez Marin J, et al. Predictive factors of suicidal behavior and suicide in adolescents and young people: a systematic review. CRD42013005775 (http://www.crd.york.ac.uk/ PROSPERO).

17 Stroup D, Berlin J, Morton S. Meta-analysis of observational studies in epidemiology: a proposal for reporting. JAMA 2000; 283: 2008-12.

18 Silverman $\mathrm{M}$, Berman $\mathrm{A}$. Rebuilding the tower of Babel: a revised nomenclature for the study of suicide and suicidal behaviors. Part 2: suicide-related ideations, communications, and behaviors. Suicide Life Threat Behav 2007; 37: 264-77.

19 Money J. The concept of gender identity disorder in childhood and adolescence after 39 years. J Sex Marital Ther 1994; 20: 163-77.

20 World Health Organization. The ICD-10 Classification of Mental and Behavioural Disorders. WHO, 1992.

21 Wells GA, Shea B, O'Connell D, Peterson J, Welch V, Losos M. The NewcastleOttawa Scale (NOS) for assessing the quality of nonrandomised studies in meta-analyses. Ottawa Hospital Research Institute, 2014 (http://www.ohri.ca/ programs/clinical_epidemiology/oxford.asp).

22 Fergusson DM, Horwood $\mathrm{U}$, Beautrais AL. Is sexual orientation related to mental health problems and suicidality in young people? Arch Gen Psychiatry 1999; 56: 876-80.

23 Skegg K, Nada-Raja S, Dickson N, Paul C, Williams S. Sexual orientation and self-harm in men and women. Am J Psychiatry 2003; 160: 541-6.

24 Wichstrom L, Hegna K. Sexual orientation and suicide attempt: a longitudinal study of the general Norwegian adolescent population. J Abnorm Psychol 2003; 112: 144-51.

25 Silenzio VMB, Pena JB, Duberstein PR, Cerel J, Knox KL. Sexual orientation and risk factors for suicidal ideation and suicide attempts among adolescents and young adults. Am J Public Health 2007; 97: 2017-9.

26 Young R, Riordan V, Stark C. Perinatal and psychosocial circumstances associated with risk of attempted suicide, non-suicidal self-injury and psychiatric service use. A longitudinal study of young people. BMC Public Health 2011; 11: 875.

27 Whitlock J, Muehlenkamp J, Eckenrode J, Purington A, Baral Abrams G, Barreira $P$, et al. Nonsuicidal self-injury as a gateway to suicide in young adults. J Adolesc Health 2013; 52: 486-92.
28 Zhang J, Yu KF. What's the relative risk? A method of correcting the odds ratio in cohort studies of common outcomes. JAMA 1998; 280: 1690-1.

29 Higgins JPT, Thompson SG. Quantifying heterogeneity in a meta-analysis Stat Med 2002; 21: 1539-58.

30 Harbord RM, Egger M, Sterne JAC. A modified test for small-study effects in meta-analyses of controlled trials with binary endpoints. Stat Med 2006; 25 3443-57.

31 Fergusson DM, Horwood L, Ridder EM, Beautrais AL. Sexual orientation and mental health in a birth cohort of young adults. Psychol Med 2005; 35 971-81.

32 Bearman PS, Moody J. Suicide and friendships among American adolescents. Am J Public Health 2004; 94: 89-95

33 Noell JW, Ochs LM. Relationship of sexual orientation to substance use, suicidal ideation, suicide attempts, and other factors in a population of homeless adolescents. J Adolesc Health 2001; 29: 31-6.

34 Shaffer $D$, Fisher $P$, Parides $M$, Gould M. Sexual orientation in adolescents who commit suicide. Suicide Life Threat Behav 1995; 25: 64-71.

35 Fried LE, Williams $\mathrm{S}$, Cabral $\mathrm{H}$, Hacker $\mathrm{K}$. Differences in risk factors for suicide attempts among 9th and 11th grade youth: a longitudinal perspective. J Sch Nurs 2012; 29: 113-22.

36 D'Augelli AR, Grossman AH, Salter NP, Vasey JJ, Starks MT, Sinclair KO. Predicting the suicide attempts of lesbian, gay, and bisexual youth. Suicide Life Threat Behav 2005; 35: 646-60.

37 Mustanski B, Liu RT. A longitudinal study of predictors of suicide attempts among lesbian, gay, bisexual, and transgender youth. Arch Sex Behav 2013; 42: $437-48$.

38 Burns MN, Ryan DT, Garofalo R, Newcomb ME, Mustanski B. Mental health disorders in young urban sexual minority men. J Adolesc Health 2015; 56: $52-8$

39 Lohonen J, Isohanni M, Nieminen P, Miettunen J. Coverage of the bibliographic databases in mental health research. Nord J Psychiatry 2010; 64: $181-8$.

40 Devries KM, Mak JY, Bacchus L, Child JC, Falder G, Petzold M, et al. Intimate partner violence and incident depressive symptoms and suicide attempts: a systematic review of longitudinal studies. PLOS Med 2013; 10: e1001439.

41 Buller AM, Devries KM, Howard LM, Bacchus L. Associations between intimate partner violence and health among men who have sex with men: a systematic review and meta-analysis. PLOS Med 2014; 11: e1001609.

42 Maxwell L, Devries K, Zionts D, Alhusen JL, Campbell J. Estimating the effect of intimate partner violence on women's use of contraception: a systematic review and meta-analysis. PLOS One 2015; 10: e0118234.

43 Holler JG, Bech CN, Henriksen DP, Mikkelsen S, Pedersen C, Lassen AT. Nontraumatic hypotension and shock in the emergency department and the prehospital setting, prevalence, etiology, and mortality: a systematic review. PLOS One 2015; 10: e0119331.

44 Daglas R, Yucel M, Cotton S, Allott K, Hetrick S, Berk M. Cognitive impairment in first-episode mania: a systematic review of the evidence in the acute and remission phases of the illness. Int J Bipolar Disord 2015; 3: 9.

45 Oremus $C$, Oremus $M$, McNeely $H$, Losier $B$, Parlar $M$, King $M$, et al. Effects of electroconvulsive therapy on cognitive functioning in patients with depression: protocol for a systematic review and meta-analysis. BMJ 2015; 5: e006966.

46 Overdevest GM, Jacobs W, Vleggeert-Lankamp C, Thome C, Gunzburg R, Peul W. Effectiveness of posterior decompression techniques compared with conventional laminectomy for lumbar stenosis. Cochrane Database Syst Rev 2015; 3: CD010036.

47 King $M$, Semlyen J, Tai SS, Killaspy $H$, Osborn $D$, Popelyuk $D$, et al A systematic review of mental disorder, suicide, and deliberate self harm in lesbian, gay and bisexual people. BMC Psychiatry 2008; 8: 70.

48 Garofalo R, Wolf RC, Wissow LS, Woods ER, Goodman E. Sexual orientation and risk of suicide attempts among a representative sample of youth. Arch Pediatr Adolesc Med 1999; 153: 487-93.

49 Botnick MR, Heath KV, Cornelisse PG, Strathdee SA, Martindale SL, Hogg RS Correlates of suicide attempts in an open cohort of young men who have sex with men. Can J Public Health 2002; 93: 59-62.

50 Paul JP, Catania J, Pollack L, Moskowitz J, Canchola J, Mills T, et al. Suicide attempts among gay and bisexual men: lifetime prevalence and antecedents. Am J Public Health 2002; 92: 1338-45.

51 Remafedi G. Suicidality in a venue-based sample of young men who have sex with men. J Adolesc Health 2002; 31: 305-10.

52 Russell ST, Toomey RB. Men's sexual orientation and suicide: evidence for U.S. adolescent-specific risk. Soc Sci Med 2012; 74: 523-9.

53 Jackson S. Gender, sexuality and heterosexuality: the complexity (and limits) of heteronormativity. Fem Theory 2006; 7: 105-21. 
54 Harter S. Self and identity development. In At the Threshold: The Developing Adolescent (eds S Feldman, G Elliott ): 352-87. Harvard University Press, 1990.

55 Pompili M, Lester D, Forte A, Seretti ME, Erbuto D, Lamis DA, et al. Bisexuality and suicide: a systematic review of the current literature. J Sex Med 2014; 11: 1903-13.

56 Machado S. The poetics of parent-son encounters following negative parental reactions to the disclosure of gay identity. J Humanist Psychol 2014 55: 30-52.

57 Meyer IH. Minority stress and mental health in gay men. $J$ Health Soc Behav 1995; 36: 38.

58 Meyer I, Dean L. Internalized homophobia, intimacy and sexual behaviour among gay and bisexual men. In Stigma and Sexual Orientation (ed. G Herek) 160-86. Sage, 1998.

59 Hammelman T. Gay and lesbian youth. contributing factors to serious attempts or considerations of suicide. J Gay Lesbian Psychother 1993; 2 77-89.
60 Armesto J, Weisman A. Attributions and emotional reactions to the identity disclosure ('coming out') of a homosexual child. Fam Process 2001; 40: 145-61.

61 Ryan $C$, Huebner $D$, Diaz $R$, Sanchez J. Family rejection as a predictor of negative health outcomes in White and Latino lesbian, gay, and bisexua young adults. Pediatrics 2009; 123: 346-52.

62 Gecas V, Libby R. Sexual behavior as symbolic interaction. J Sex Res 1976 12: $33-49$.

63 American Psychological Association. The Guidelines for Psychological Practice with Lesbian, Gay and Bisexual Clients. APA, 2011

64 O'Connor D, Green S, Higgins JPT. Defining the review question and developing criteria for including studies. In Cochrane Handbook for Systematic Reviews of Interventions (eds J Higgins, S Green): ch 5. Cochrane Collaboration, 2008.

65 Sterne J, Sutton A, Ioannidis J, Terrin N, Jones D, Lau J, et al. Recommendations for examining and interpreting funnel plot asymmetry in meta-analyses of randomised controlled trials. BMJ 2011; 342: d4002.

\title{
reflection
}

\section{The Psychopathology of Everyday Life, Sigmund Freud}

\author{
Jeremy Holmes
}

Joyous is not a word normally associated with Freud despite being the true meaning of his name. But The Psychopathology of Everyday Life fully deserves the epithet: a glorious collection of anecdotes, spoonerisms, lacunae, 'speech blunders' and odd actions - revealing, he argues, the cauldron of repressed feelings lurking below consciousness.

The book belongs to Freud's middle period, while still a passionate clinician reveling in free-associationism, and relatively unencumbered by meta-psychology. Written in 1901, published in 1904, it was first translated into English by Brill in 1914 'Fehlleistungen' was changed by Strachey from Brill's 'faulty action' to the pseudo-medical 'parapraxis'. The 'Freudian slip', with its double entendre of a revealing/concealing female undergarment, appeared in 1959.

The Psychopathology of Everyday Life reveals Freud at his most scintillating. Many examples come from his own life, professional and personal. We learn of his marital tiffs; his daughter's life-threatening illness; sexual attraction to a younger woman; omitting to pay bills and losing his keys; rivalry with colleagues; and, despite his famed photographic memory, forgetting the name of a famous Renaissance painter, Signorelli. He touchingly exposes his professional failures: an analysand's suicide; patients he mis-diagnosed with 'hysteria' who had a brain tumour or multiple sclerosis

Freud's mission is threefold: (1) to illustrate the continuity between normality and pathology, counteracting the prevalent view of mental illness as manifestation of 'degeneracy'; (2) following The Interpretation of Dreams, to show that 'dream-life' continues during the day: (3) to convince that 'faulty action' - including speech - is 'motivated' by warded-off affective states: 'forgetting in all cases is proved to be founded on a motive of displeasure'. It is not the feelings in themselves that are 'displeasureable'. Sexuality, and the desire for fame and recognition, are no less unconsciously 'motivating' than resentment, shame and disappointment. Displeasure lies in the anxiety associated with such feelings. Parapraxis arises out of what we now see as 'affect phobia'.

But was Freud right? True, scientific curiosity does not shy away from the everyday. Darwin learned as much from the worms in his Down House garden as from Galapagos finches. Freud argues for everyday material's 'admission' to science, subject to 'stricter methods of verification'. He is in awe of 'the great Darwin's' practice of noting examples which ran counter to his theories, fearing that wish-fulfillment would otherwise suppress them. Sadly, Freud fails to apply these strictures to himself. There are few if any black swans in The Psychopathology of Everyday Life; psychic determinism rules.

We know now that many slips are unmotivated, flowing from 'banalisation', verbal typos and ingrained habit. But parapraxes are also undeniable. What primary school child has, to their horror, not called mistakenly their teacher 'Mum'? Male subjects given verbal tasks by scantily clad female psychologists tend to produce unwitting sexual innuendos. Avoidantly attached individuals have high cortisol levels, suggesting suppressed attachment needs; when subject to 'cognitive load', for instance in Stroop tests, the need for security parapraxically reveals itself

A century on, Freud's ode to psychoanalytic joy has lost none of its freshness and relevance: essential reading for all would-be wise and witty psychiatrists 